\title{
Le genre encadré
}

Ethnographie d'une formation au leadership pour aspirant'e's cadres

Framed Gender. Ethnography of an Organization Aiming at Developing

Relational Skills for Aspiring Managers

\section{Hugo Wainsztok}

\section{OpenEdition \\ Journals}

Édition électronique

URL : http://journals.openedition.org/travailemploi/7700

DOI : 10.4000/travailemploi.7700

ISSN : $1775-416 \mathrm{X}$

\section{Éditeur}

DARES - Ministère du Travail

\section{Édition imprimée}

Date de publication : 1 juillet 2017

Pagination : 53-73

ISSN : 0224-4365

\section{Référence électronique}

Hugo Wainsztok, «Le genre encadré », Travail et Emploi [En ligne], 151 | juillet-septembre 2017, mis en ligne le 11 juillet 2019, consulté le 12 septembre 2019. URL : http://journals.openedition.org/ travailemploi/7700 ; DOI : 10.4000/travailemploi.7700 


\title{
Le genre encadré
}

\section{Ethnographie d'une formation au leadership pour aspirant.e.s cadres}

\author{
Hugo Wainsztok*
}

\begin{abstract}
Dans les débats sur la transformation du groupe des cadres, de nombreuses études de la littérature de gestion indiquent qu'un « nouveau capitalisme » enjoindrait à passer d'un mode de management fondé sur l'autorité et la hiérarchie à un management centré sur la coopération interindividuelle et l'animation de réseaux. L'article se propose de confronter ce corpus gestionnaire à l'évolution des savoir-faire relationnels maitrisés par les cadres, à partir de l'ethnographie d'une association parisienne promouvant l'apprentissage de compétences au leadership. En croisant les dimensions de genre et de classe, l'enquête montre que ces compétences n'aboutissent pas à une meilleure promotion des femmes, comme l'écrivent les théoriciens du management. Les pratiques promues par l'association se trouvent réappropriées par les membres, qui développent ainsi des pratiques diversifiées. Elles contribuent, comme nos observations le mettent en évidence, à asseoir la domination de certains profils d'hommes.
\end{abstract}

L e modèle de l'autorité paternaliste connaît une profonde remise en cause dans les écrits managériaux depuis les années d'après-guerre en France. Dans l'univers des sciences de la gestion, qui a été repris par le syndicalisme patronal plus tardivement ${ }^{1}$, de nombreux chercheurs constatent l'arrivée en force d'une série de discours volontaristes pour une plus grande autonomie des salarié.e.s et d'une transformation des relations hiérarchiques en faveur d'une « cité par projet », combinant la construction de « réseaux » et une structure de travail en projet (BOLTANSKI, ChiAPELlo, 1999).

\footnotetext{
* Institut de recherche interdisciplinaire sur les enjeux sociaux (Iris), École des hautes études en sciences sociales (EHESS) et CNRS ; hugo.wainsztok@ehess.fr.

1. En 1997, la nouvelle vision entrepreneuriale présentée par le Medef symbolise la rupture avec l'ancien CNPF. La doxa entrepreneuriale de l'autonomie et de la liberté d'entreprendre rejoint un glissement sémantique : «patron » devient «entrepreneur », et le terme d' « entrepreneur » vient s'opposer à «l'establishment» et aux « hommes d'appareil ».
} 
Ces discours visent à transformer les pratiques d'encadrement par la valorisation de compétences dites relationnelles et par la délégation des tâches de coordination et de contrôle à des dispositifs automatisés. Dans le monde du travail, nous voyons donc apparaître un aggiornamento des techniques du pouvoir, qui invite les managers à modifier leurs subjectivités, à se montrer en entreprise plus attentifs aux autres, et à soutenir le développement personnel de leurs subordonné.e.s (LiNHART, 2003 ; SALMAN, 2014 ; BorZEIX et al., 2015).

De telles évolutions ne sont pas neutres du point de vue des inégalités entre femmes et hommes, et méritent d'être analysées sous l'angle du genre ${ }^{2}$, tant la féminisation du groupe des cadres invite des directions d'entreprises, appuyées par des entrepreneuses de morale (BLANCHARD et al., 2013), à associer horizontalité du pouvoir et performance économique avec la diversité des sexes en entreprise (AMINTA, JunTER, 2009). Selon cette conception, les femmes apporteraient plus de coopération et/ou des styles de commandement plus doux. La présence accrue de femmes cadres serait vecteur de modes de direction plus consensuels, favoriserait une meilleure coopération de travail entre salariés et serait un gage de productivité (LAUFER, 2009 ; BERENI, 2011). Par certains côtés, cette position rejoint les thèses des féministes différentialistes, qui proposent de lutter contre l'androcentrisme par la défense et la promotion de qualités « féminines », pensées comme propres aux femmes ${ }^{3}$ (BILling, Alvesson, 1989). Cette vision positive du management au féminin se heurte néanmoins à des inégalités qui en contrarient la portée. Si la proportion de femmes parmi les cadres a augmenté en France, puisqu'elles représentent $41,6 \%$ de cette catégorie contre $30,8 \%$ il y a vingt ans ${ }^{4}$, leur présence reste très inégale selon les domaines d'activité. Le progrès observé dans l'accès des femmes à la catégorie cadre est surtout porté par les secteurs de l'administration publique, de l'enseignement, de la santé et de l'action sociale, et moins par le secteur de l'agriculture, de la construction ou de l'industrie. Malgré des dispositions législatives récentes en faveur de quotas pour les conseils d'administration du privé et du public ${ }^{5}$, on observe que plus un poste offre un haut niveau de salaire et un prestige social élevé, moins les femmes y sont représentées. Dans les entreprises privées de 500 salarié.e.s ou plus, les femmes n'occupent que $23 \%$ des fonctions de cadres dirigeant.e.s alors qu'elles représentent la moitié des salarié.e.s du privé ${ }^{6}$. Un

2. La notion de genre est entendue dans l'article comme un réservoir de « symboles culturellement disponibles » et de concepts normatifs intériorisés par les individus, de façon toutefois variable dans le temps et l'espace social (ScoTT, 1986). Selon cette acception, le genre n'est une catégorie d'analyse qu'une fois articulé à d'autres variables, telles que le groupe ethno-racial ou la classe sociale des enquêtés.

3. Dans les années 1980, plusieurs féministes différentialistes insistent sur la complémentarité des sexes et la valorisation de la spécificité de valeurs, de sentiments et de comportements féminins : «This women-centrered perspective celebrates and exonerates female difference, instead of suggesting that women imitate male agenic features with an androgynous sprinkling of communal qualities » (GRANT, 1988, citée par BILLING, ALVESSON, 1989, p. 71).

4. Voir InsEe, 2017.

5. Loi relative à la représentation équilibrée des femmes et des hommes au sein des conseils d'administration et de surveillance et à l'égalité professionnelle (27 janvier 2011) et Loi pour l'égalité réelle entre les femmes et les hommes (4 août 2014).

6. Voir INSEE, 2017. 
tour d'horizon des recherches concernant les inégalités hommes-femmes chez les cadres met en évidence les effets de la « conciliation » entre travail domestique et travail professionnel (PAILHÉ, SOLAZ, 2010), ainsi que le plafond de verre et la ségrégation sexuée des emplois (LAufer, 2005 ; Pochic, Guillaume, 2007) ; la dimension organisationnelle des discriminations selon le sexe renvoie notamment à l'orientation des femmes vers les postes d'experte et de cheffe de projet/mission plutôt que sur les métiers techniques d'ingénieures (MARRY, 2001) ou d'encadrement (PochIC, 2005). En revanche, la compréhension des savoir-faire relationnels en situation de mixité a

\section{ENCADRÉ 1}

\section{Une enquête ethnographique dans une association parisienne de Becomealeader}

Les analyses présentées dans cet article sont issues d'une enquête ethnographique menée de décembre 2012 à avril 2013 dans deux clubs parisiens à but non lucratif. Ces deux structures se réclament d'une même association internationale renommée ici Becomealeader, comme dix autres clubs dans Paris et sa banlieue. L'association est née aux États-Unis en 1924. Elle a été conçue par un proche de l'Église presbytérienne, responsable de la Young Men's Christian Association dans l'Illinois ${ }^{1}$. Sans lien confessionnel à l'heure actuelle, ce modèle atteint l'Hexagone à la fin des années 1980, en étant revisité par des cadres français. Alors qu'aux États-Unis il est intégré aux grandes entreprises, son introduction en France se fait sous la forme d'associations à but non lucratif, hors de l'entreprise, après une tentative infructueuse 2 .

Le présent article se focalisera sur l'un des deux clubs, dont les activités ont lieu dans la salle polyvalente d'un centre d'animation de la Ville de Paris. J'ai pu y observer une dizaine de séances de formation à découvert. En me présentant comme un étudiant en sociologie, j'ai dans un premier temps eu le statut d'invité dans le club. Puis c'est en tant qu'adhérent, suite au paiement d'une cotisation de trente-cinq euros, que j'ai effectué mes dernières observations. Cette adhésion, un mois après ma première venue en séance, m'a permis d'approcher au plus près les acteurs. Elle m'a obligé à intervenir un minimum lors de chaque séance, comme l'ensemble des participants. Le temps passé assis à écouter les interventions des membres m'a donné la possibilité d'une prise de notes aisée. En tant que nouvel adhérent, j' ai aussi reçu deux manuels (Savoir communiquer et Devenir un Leader) ainsi que d'autres ressources numériques envoyées par les membres du bureau par courriels, ensemble documentaire que j'ai intégré à l'analyse, notamment les manuels Pour la gestion efficace du club et Évaluation efficace. Simultanément, j'ai réalisé plusieurs entretiens semi-directifs (neuf au total) auprès de participant-e.s régulier.e.s afin de connaître leurs parcours et de comprendre leur manière de s'emparer du discours managérial prodigué par l'association.

1. Informations disponibles sur le site internet de l'association internationale Becomealeader (le nom de l'organisme a été volontairement modifié).

2. «Un club d'entreprise forcément tu apprends des choses. Ton supérieur sait que tu y passes du temps et il a envie que tu t'y investisses vraiment. On a déjà eu un club d'entreprise francophone, qui n'a pas fonctionné. Parce que les gens étaient obligés d'y aller, et ça n'a pas marché. » Entretien avec Didier en avril 2013. 
peu été étudiée (STEVENS, 2013), alors même que les années 1990 ont vu s'accroître le nombre de dispositifs de formation visant spécifiquement la prise en compte de ces savoir-faire dans la sphère professionnelle et extra-professionnelle.

L'article souhaite confronter les conceptions gestionnaires à la réalité des savoirfaire relationnels maîtrisés par les cadres, en se fondant sur une enquête de terrain au sein d'une association de formation au leadership (encadré 1). La première partie présente les activités proposées par cette association et décrit les cadres qui y adhérent, que le sens qu'ils donnent à leur apprentissage. La deuxième partie détaille l'effet des propriétés sociales des adhérents sur les savoir-faire promus par le club. Elle établit que les différences de genre et de classe s'accompagnent d'appropriations différentes de la figure du leader et se traduisent par des formes de retrait pour les femmes et par une domination euphémisée chez les hommes. Enfin, par le biais des exercices oratoires réalisés par les adhérent.e.s, la dernière partie montre comment les capacités langagières et de présentation de soi sont inégalement assimilées chez les hommes. Ces capacités nous semblent valoriser davantage les profils d'hommes issus de la partie haute des classes moyennes, pouvant atténuer leur position sociale élevée, et contribuent à discréditer les membres d'origine plus populaire.

\section{Un lieu d'ajustement pour salariéee's non manuel·le's et aspirant·e's cadre}

\section{Le dispositif}

Au sein de l'association, l'amélioration du leadership et des compétences en communication est censée reposer sur le travail d'équipe. L'organisation des séances, autogérée par les participant·e·s, vise ainsi à affaiblir l'asymétrie des relations liées à la situation d'apprentissage en prévoyant la distribution de rôles différents à chaque séance (encadré 2). Dans les manuels de l'association il est écrit à ce sujet : « La mission du club est d'offrir un environnement d'apprentissage dans lequel les participants s'encouragent mutuellement de façon positive afin de permettre à chacun d'améliorer ses capacités de communicants et de leaders, permettant de générer confiance en soi et développement personnel. » Les réunions et les textes - littérature grise et manuels - produits ou traduits de l'anglais par les adhérent.e.s diffusent une norme d'internalité qui rappelle les thérapies de groupe ou les dispositifs de « l'entreprise de soi » (STEVEns, 2012). Les allusions et termes psychologisants, tels que le « développement personnel » ou le « potentiel humain », fixent une conception de l'individu comme défait de ses déterminismes sociaux, possédant un potentiel et des capacités relationnelles. Le slogan de l'association - « devenez le leader que vous souhaitez être »- résume cette orientation en usant d'une rhétorique à la fois méritocratique et vocationnelle. Malgré cela, l'espace de socialisation professionnelle que constitue le club se distingue des séminaires de développement personnel : il prévoit un cadre plus 


\section{ENCADRÉ 2}

\section{L'organisation d'une séance du club}

Une séance réunit une trentaine de participant·e-s qui s'attribuent des rôles. Le terme « rôle » fait référence au théâtre d'improvisation, dont le dispositif s'inspire. En attestent les quatre coups de marteau donnés sur le pupitre par le Président de séance à chaque début de réunion.

Différents rôles sont distribués à chaque séance aux participants. Aucun membre ne peut endosser le même rôle pendant plusieurs séances. Ces rôles supposent de leurs détenteurs et détentrices l'exercice d'une activité fonctionnelle pour le collectif, comme le contrôle (avec le Chronométreur, les Évaluateurs, etc.), la présentation (le Meneur des improvisations, etc.), la coordination (le Président de séance). Tou·te·s les membres s'entraînent par ailleurs lors des séances à la pratique orale et organisationnelle à titre plus personnel.

Les séances se construisent autour d'un planning administré par le Président de séance, toujours structuré de la même manière, en quatre séquences : 1 . les discours préparés ; 2 . les improvisations ; 3 . une pause ; 4. les évaluations. Un temps strict est prévu pour chaque étape. Le rôle du Chronométreur est de surveiller le temps de chaque passage à l'aide d'un chronomètre. Si un·e intervenant.e ne va pas assez vite, il l'arrête en brandissant un panneau rouge. La comptabilisation du temps s'applique aussi bien aux interventions, qui peuvent durer de 2 à 7 minutes, qu'aux pauses (5 à 10 minutes maximum).

Les séances respectent un placement spécifique du mobilier. Des tables rectangulaires sont placées pour former un U, avec au centre un pupitre en bois. Les chaises sont installées face aux tables, de façon à ce que l'ensemble des individus ait vue sur le pupitre où s'expriment les orateurs et oratrices. Un marteau de juge posé sur le pupitre complète le décor.

formalisé, tourné vers la prise de parole en public et la communication au sein d'un dispositif collectif mais individualisé, par l'entremise d'objectifs intitulés « projets ».

\section{Les membres réguliers du club : souhait d'une promotion sociale et déficit de capital culturel}

Du fait du caractère libre et non lucratif de la participation, et de la gamme relativement large des apprentissages qui va de la confiance en soi à la maîtrise de la communication et au leadership, le club attire un public hétérogène : étudiant·e.s, salarié·e·s du secteur privé, fonctionnaires, retraité·e·s, chômeurs et chômeuses ainsi que dans une plus large mesure des commerciaux et commerciales, et des consultant.e.s. Ma participation sur plusieurs mois m'a permis toutefois de distinguer les membres réguliers des participants plus ponctuels. Le public régulier se rapproche de manière plus ou moins marquée d'un profil de salarié non manuel issu des « nouvelles classes moyennes » (GouX, MAURIN, 2012). On trouve notamment des fils et filles d'artisan·e·s 


\section{ENCADRÉ 3}

\section{Profil des membres réguliers du club ${ }^{1}$}

Sur les onze membres réguliers, quatre sont des femmes, contre sept hommes ; cinq ont le statut de cadre.

Audrey - 34 ans, assistante commerciale dans une entreprise de la grande distribution, licence d'AES à l'université, membre depuis deux ans ;

Annie - 55 ans, cadre de santé au CHU, études d'infirmière, membre depuis sept ans ;

Boris - 33 ans, chef de projet, ingénieur en sécurité informatique, diplômé de l'École centrale de Paris, membre depuis sept ans ;

Carole - 36 ans, juriste en entreprise, D.E.S.S Droit et pratique de l'assurance à l'Université de Bordeaux, membre depuis un an ;

Cédric - 39 ans, informaticien/consultant en entreprise, licence d'informatique à De Montfort University, « petite » université anglaise, membre depuis un an ;

Déborah - 42 ans, employée de banque, diplômée d'un baccalauréat, nationalité italienne, membre depuis 6 mois ;

Didier - 51 ans, cadre commercial dans une entreprise de la grande distribution, diplômé d'une « petite » école de commerce, membre depuis dix ans ;

Mickaël - 25 ans, sans emploi, cherche du travail dans la publicité ou envisage de monter une start-up, licence en communication à l'université la Sorbonne, membre depuis deux ans ;

Jean - 32 ans, coach en soft skills, diplômé d'une «petite » école de commerce (l'ESCEM), membre depuis trois ans ;

Kevin - 43 ans, commercial dans une entreprise de location de bureaux, diplômé de l'ESC Montpellier, membre depuis six mois ;

Victorien - 38 ans, dentiste/entrepreneur dans la santé, études d'odontologie, membre depuis deux ans.

1. Afin de préserver l'anonymat des personnes ayant participé à l'enquête, les noms des différents acteurs et actrices ont été modifiés.

et de commerçant·e·s à faibles ressources culturelles, qui ont profité de l'ouverture de l'enseignement supérieur des années 1990, permettant à plus de la moitié des bachelier·e.s issu·e.s de classes intermédiaires et supérieures de poursuivre leurs études. Les participant·e·s se démarquent des classes populaires par une socialisation scolaire longue, mais possèdent presque tous des diplômes peu valorisés par rapport à leur statut professionnel actuel ou souhaité. Ils et elles sont diplômé·e·s, pour la grande majorité, d'écoles de commerce situées en province ou d'universités. Si ces diplômes leur permettent d'occuper un emploi de cadre - 65 \% des diplômé·e·s d'école de commerce en 2004 sont cadres en 2007 (CALMAND et al., 2009) -, leur prestige est très variable et sortir d'une « petite » école est un puissant stigmate au sein des grandes entreprises (PoCHIC, GuILlaume 2007). Cette morphologie sociale se retrouve dans le 
deuxième club étudié, mais se distingue de celle des membres des clubs Becomealeader anglophones ou du club franco-allemand, qui s'adressent à une population davantage diplômée et polyglotte ${ }^{7}$.

Dans cette formation à bas coût ${ }^{8}$, les membres régulier-e.s se distinguent des participant·e-s plus ponctuel-le·s par la fréquentation par ailleurs de séminaires de développement personnel, qui les rendent réceptifs et réceptives aux vocabulaires psychologisants du dispositif ${ }^{9}$. L'adhésion des membres à ces valeurs est vérifiée à la fin de chaque meeting où, selon l'usage, le Président demande aux nouveaux arrivants et nouvelles arrivantes, appelé.e.s « invité.e.s », de se présenter et de donner leur avis sur la séance écoulée. Les invité.e.s qui disent ne pas comprendre ou qui trouvent l'organisation trop rationnelle ou insolite, ne reviennent pas ou peu aux réunions. Les adhérent·e.s régulier·e·s partagent l'idée qu'ils et elles souffrent à des degrés divers d'une mauvaise communication orale, de difficultés relationnelles et d'un manque de confiance en eux et en elles ; défauts que Boris (33 ans, chef de projet/ingénieur informatique) me décrit comme « rédhibitoires dans un monde de communication». En dehors des réunions, il arrive qu'ils expriment sur le ton de l'humour leur stress face aux incertitudes du marché du travail et aux pressions des clients. C'est pourquoi, pour lutter contre la stagnation de leur situation professionnelle ou bien pour faciliter le quotidien dans leur emploi, ces salarié.e.s aspirant à une position de cadre ou d'encadrement s'accordent tou·te·s pour travailler leur savoir-être.

\section{Un apprentissage de pratiques socialement sélectives}

Le leader est conçu par l'association comme prioritairement doté d'entregent : il « stimule et encourage », il « coordonne et régule les activités du groupe » et « crée un environnement de travail serein en encourageant les membres $»$. Le manuel numérique Pour la gestion efficace du club ajoute que les leaders doivent accorder une attention prioritaire aux besoins des autres membres : « le leadership est un voyage permanent qui comprend le désir de servir les autres et l'engagement à diriger : efforcez-vous d'être digne de confiance, conscient de soi, humble, optimiste, compatissant et stimulant ». De manière cohérente avec cette aversion pour la figure du chef, on distingue sur la même page du manuel le patron du leader : "Soyez un leader et non un patron, votre travail est d'aider les leaders du club à atteindre leurs objectifs de formation ${ }^{10}$. »

\footnotetext{
7. Onze clubs se réclament du modèle Becomealeader dans Paris et sa banlieue. Si dans la majorité d'entre eux la langue utilisée est le français, à Paris, il existe aussi un club franco-allemand et trois clubs anglophones.

8. La cotisation à l'année est de 70 euros, et de 35 euros pour 6 mois. Elle sert essentiellement à payer l'impression des plannings, des fiches d'évaluation et les manuels de l'association Becomealeader.

9. En entretien, les membres régulier·e·s ont en commun d'avoir tous suivi un séminaire de développement personnel, sauf pour Mickaël et Carole.

10. Cette définition du leadership rappelle les développements des premier·e·s théoricien·ne·s en management à l'instar de Mary Follett (1868-1933), qui considérait que «chaque homme tend à avoir le leadership que son travail particulier lui confère, ce qui est vrai pour le président comme pour tous les autres ». Dans un texte intitulé «Leader and Expert » elle ajoute : «L'un des principaux devoirs du leader est d'extraire de chacun ses pleines possibilités. [...] Le leader n'est pas le boss, mais l'éducateur. »(COHEN, 2013, p. 393).
} 
Cette conception du leader euphémise les conduites autoritaires et valorise la bienveillance et le renforcement du contrôle des «façades personnelles ${ }^{11}$ » chez les participant.e.s. Cela s'observe par la mise en scène de la proximité et d'affects joyeux, dont beaucoup de descriptions font état, comme j'ai pu l'observer lors des séances :

«Les membres se saluent puis commencent à discuter entre eux en se tutoyant. À l'extrémité de la salle un groupement s'est formé autour d'une table sur laquelle sont installés à disposition de tous des produits apéritifs (...). On a l'impression d'une fête entre amis. »

(Carnet de terrain, séance du 10 janvier 2013)

Mon impression personnelle est confirmée par plusieurs membres. Cédric (39 ans, informaticien en entreprise) me dit en séance : «Ce qui est bien ici, c'est l'ambiance vraiment sympa... » Ou encore, une nouvelle adhérente déclare aimer particulièrement l'ambiance chaleureuse et la coopération mutuelle. En attestent aussi les présentations que font d'eux et d'elles les membres, qui se montrent toujours plein d'entrain et d'optimisme. Les propos de plusieurs enquêté.e.s témoignent de l'état de satisfaction, voire de joie du participant. En outre, ils n'hésitent pas à se féliciter quand une présentation orale contient de l'émotion, des sentiments, de l'humour ou des mots positifs. Annie (55 ans, cadre de santé) insiste régulièrement sur le fait qu' une prestation orale est réussie quand il y a de l'humour. Dans un extrait de discours prononcé en tant qu'Évaluatrice (encadré 1), elle s'exclame : « L'humour, cela permet de faire plaisir au public et de garder une ambiance agréable et enthousiaste. Mes amis, manier l'humour c'est très important pour les leaders que nous sommes ! Même constat avec l'utilisation courante du terme « soirée » plutôt que « réunion » ou « séance » qui évoquent plus directement le travail. Cet esprit se retrouve dans les évaluations adressées aux nouveaux inscrits. Ainsi, Éric, cadre d'une trentaine d'années, nouveau dans le club, dit à Fabienne, retraitée de la fonction publique et nouvelle elle aussi :

« J'ai bien aimé ton discours, tu l'as bien enjolivé avec des mots positifs ; j' ai entendu «parcours très formateur », « motivation », « enthousiasme ». Ce sont les mots que tu as utilisés en général dans ton discours et c'est très bien. »

Entretenir une présentation de soi convenable est d'autant plus important pour les adhérents que ne pas jouer le jeu peut être passible d'exclusion. C'est ce que j' apprends en discutant avec Didier (51 ans, commercial) inscrit dans l'association Becomealeader depuis dix ans. Selon lui, une adhérente a déjà été exclue du collectif à cause de son incapacité à développer, même un minimum, cet entregent :

«Y a-t-il déjà eu des invités qui n'ont pas eu accès au statut de membre dans le club ?

11. «[...] pour désigner les éléments qui, confondus avec la personne de l'acteur lui-même, le suivent partout où il va. On peut y inclure : les signes distinctifs de la fonction ou du grade ; la taille et la physionomie ; l'attitude ; la façon de parler ; les mimiques ; les comportements gestuels et autres éléments semblables. » (GoFFMAN, 1973, p. 30). 
- Non, mais... si, si... il y en a eu une, que je trouvais trop timide, pas assez sociable, elle ne jouait pas le jeu. »

(Carnet de terrain, séance du 4 avril 2013)

Du point de vue des organisations productives, ces savoir-faire interactionnels peuvent participer à la pacification des relations de travail ${ }^{12}$. D'un autre côté, il est aussi une partie intégrante de l'ethos professionnel des commerciaux ou commerciales et des consultant·e·s, professionnel-le·s qui composent la moitié des effectifs du club. Les observations montrent que la valorisation de la notion de leader s'apparente à l' aisance dans le maniement de la parole et à l'apprentissage d'une familiarité avec les strates supérieures de la bourgeoisie. Le moment de pause au milieu des séances est à ce titre particulièrement éclairant, puisqu'un·e participant·e choisi-e par le Président de séance est sommé·e de « porter un toast », c'est-à-dire d'improviser un discours en présence des membres avant de partager l'apéritif. L'acquisition de ces façons d'être peut se révéler décisive dans le milieu patronal ou en entreprise. Elles servent de «capital culturel non certifié » (BERNARD, 2012), pouvant participer à la création d'un réseau d'interconnaissance, puis être mis à profit en vue d'une ascension professionnelle.

\section{Être leader : vers une figure renouvelée du chef}

\section{Épanouissement personnel pour les femmes, formation au leadership pour les hommes}

Lors des séances, les hommes et les femmes subissent un contrôle vestimentaire inégal. Les adhérent·e·s ne sont pas nécessairement vêtu·e·s d'une veste, comme signalé lors de ma première participation : «Tu sais, tu n'es pas obligé de venir tout le temps avec ton costume. » En revanche, le port d'une chemise simple, souvent déboutonnée, est privilégié par les participants masculins ${ }^{13}$. Sans que ce soit spécifique au club, cette pratique différencie les hommes, en chemise, des femmes, jouissant manifestement d'une plus grande latitude vestimentaire. Une polarité entre les sexes existe également dans le sens donné à l'apprentissage. Si la plupart des membres utilisent le terme de « relationnel », la notion ne renvoie pas à la même réalité. Ainsi, les femmes participant régulièrement aux séances justifient leur investissement en arguant

\footnotetext{
12. Le texte numérique Évaluation efficace, fourni à chaque participant, regorge de techniques rhétoriques visant à affaiblir la portée d'une évaluation ou d'un jugement au sein d'un environnement de travail. C'est le cas de l'utilisation de « la méthode sandwich », qui consiste à proposer les idées d'amélioration entre deux commentaires positifs. Il est demandé à l'évaluateur ou l'évaluatrice de ne pas faire usage du « nous » ou « il », pour ne pas inclure les adhérent·e·s dans la critique. Il est recommandé d'éviter les phrases péremptoires comme « un bon leader ne fait jamais... ». D'autres conseils permettent de maintenir une ambiance agréable. Si l'évalué·e est mécontent·e de l'évaluation, le manuel précise : «Demander des explications à l'évaluateur, mais seulement en tête-à-tête ».

13. Cette manière de s'habiller ne marque pas un décalage complet avec le monde du travail, mais évoque plutôt un cadre décontracté.
} 
d'une volonté d'épanouissement personnel comme objectif principal, au détriment de l'apprentissage du leadership, surtout mis en avant par les hommes. La majorité des femmes membres disent venir aux séances pour perdre leur timidité et améliorer leur habileté à communiquer. Elles insistent sur l'apprentissage de la communication plutôt que sur la notion de leader - ce mot est plus rarement employé en entretien par les adhérentes régulières (Audrey, Carole et Déborah) que par leurs homologues masculins. L'association entérine de fait la disjonction entre communication et leadership à travers les deux manuels qu'obtient chaque participante après le paiement d'une cotisation : Savoir communiquer et Devenir un leader.

Les motivations mises en avant par les hommes sont différentes. Au contraire des femmes, leur discours couvre, à de rares exceptions près, l'ensemble des apprentissages prodigués au sein du club. Il ne dissocie ainsi pas le travail psychologique et la confiance en soi de la formation au leadership ; étant entendu qu' un bon communicant est un leader et qu'un leader est un être accompli au travail. Les adhérents se réfèrent tous à des manques ou à des blocages, qui les obligeraient à un travail sur eux-mêmes avant ou en parallèle de la formation au leadership. En témoigne par exemple un extrait d'entretien avec Boris (33 ans, ingénieur) :

«Un des premiers trucs que tu apprends en développement personnel c'est qu'il faut que tu sois physiquement et mentalement en forme déjà, avant de pouvoir te préoccuper de ce que tu crois être tes problèmes. Si tu n'as pas l'énergie qu'il faut, tu ne vas pas pouvoir atteindre tout ton plein potentiel pour les autres problèmes au boulot, donc c'est cohérent en fait. »

(Entretien réalisé en février 2013)

La distinction entre sexes apparaît avec la réinterprétation par les adhérent·e.s de la notion de leader et des savoir-faire interactionnels qui lui sont associés. En dehors des discours, des pratiques différenciées s'observent aussi entre les hommes et les femmes dans la gestion du travail associatif. Toutes les tâches permettant la préparation des réunions sont systématiquement effectuées par les hommes : imprimer les plannings, amener et disposer le matériel requis, planifier les réunions en contactant les cotisants par courriel, tenir le calendrier du club et se charger de son budget. Ceux qui assument ces tâches - les membres du bureau ${ }^{14}$, qui sont ici des hommes - ont tendance à les valoriser en invoquant leur caractère vital pour le groupe. L'effet combiné des normes masculines et de dispositions sociales relevant de la classe moyenne semble induire chez les adhérents hommes un devoir d'implication supérieur en séance. Leur « problème de communication », considéré comme une «barrière à la réussite », constitue un tel enjeu pour eux qu'ils s'investissent à plein. Inversement, la position de retrait des adhérentes se trouve certainement exacerbée sur le terrain par le nombre supérieur d'hommes parmi les membres régulier.e.s - sept sur onze, contre une trentaine de participant·e.s plus irrégulier.e.s à chaque séance. Cette situation m'est peut-être

14. Tous les clubs désignent un bureau, dont les membres ont pour fonction de préparer les réunions. Leur mandat dure un an à l'issue d'un vote à la majorité des participant·e·s du club. 
apparue plus fortement en raison de la situation d'enquête : bien que ma position de participant novice ait pu me rapprocher d'elles, mon statut d'enquêteur masculin a sans doute renforcé les attendus et différences de genre.

\section{«Que fait Hillary Clinton pour s'amuser $?^{15}{ }^{\text {» }}$ le travail d'interaction capté par les hommes}

Dans le manuel intitulé Savoir communiquer, il est écrit à la première page : « Le programme de l'association n'est ni une Université, ni une École de commerce, ni un autre cours formel de prise de parole en public. Vous n'y trouverez ni instructeurs, ni professeurs, ni salle de cours. Votre travail ne sera pas noté et vous ne passerez pas d'examen. » L'apprentissage s'effectue uniquement grâce à des manuels individuels et à une pédagogie de type learning by doing. Cette méthode, développée dans l'association au plan international, consiste à former des leaders à partir de situations jouées en séance. Elle repose sur l'idée que l'activité en club est un outil de développement de soi au service de la formation. L'entrée de nouveaux arrivants et nouvelles arrivantes dans ce type de dispositif a des répercussions sur le déroulé des séances. L'absence d'explication, conséquence de cette forme de pédagogie, place les nouvelles recrues dans une position d'inconfort; il arrive d'ailleurs que quelques-un.e.s l'expriment ouvertement :

«La réunion se termine par les mots de clôture de Mickaël le Président de séance, qui demande ensuite, comme à chaque fois, aux invités leur avis sur la séance. Une invitée prend la parole : "Je n'ai rien compris à l'organisation, pouvez-vous rendre tout cela plus explicite ?" Mickaël élude la question : "D'accord, je vous conseille de revenir à la prochaine séance pour mieux comprendre".»

(Carnet de terrain, séance du 22 février 2013)

Ce témoignage n'est pas un cas isolé. Il dit bien l'incompréhension des invitée-e·s face au dispositif. Il arrive fréquemment, au cours des réunions et surtout lors des pauses, que de nouveaux participants ou nouvelles participantes sollicitent l'aide de membres plus expérimenté.e.s. C'est ce qu'indique Cédric (39 ans, informaticien en entreprise) lors de son discours d'accueil pour l'intronisation d'un nouvel adhérent·e. Pour lui, malgré une pédagogie et une organisation très structurées, les règles sont « ambiguës », « troubles ». Il suggère en conséquence aux arrivant.e.s ou membres invitée.es de ne pas hésiter à solliciter des leaders aguerri.e.s. Or, lors des situations d'incertitude, ce sont systématiquement les hommes qui prennent l'initiative d'aider ou d'encourager les nouveaux ou les nouvelles arrivant.e.s. Si bien que les femmes

15. David Brooks, dans un éditorial du New York Times daté du 24 mai 2016 s'interroge : « Pourquoi Hillary Clinton est-elle si impopulaire ?» Puis il continue : « Je commencerai mon explication par cette interrogation : pouvez-vous me dire ce que fait Hillary Clinton pour s'amuser ? ». Ces propos recueillis par John R. MacArthur dans Le Monde diplomatique lient la légitimité politique avec le fait de s'amuser. Ils rejoignent en cela l'idée qu' une position d'autorité, pour être légitime, devrait passer par la sympathie afin d'euphémiser le pouvoir qui lui est attaché. John R. MacArthur, « Union forcée autour de Hillary Clinton », Le Monde diplomatique, août 2016. 
adhérentes sont rendues peu visibles, par la constitution d'une sorte de division des rôles :

« Juste après m'être assis, j'observe Victorien. Il parle à l'Italienne aux cheveux courts, nouvelle venue au club : "Ne t'en fais pas, ça va bien se passer !" Tout au long de la séance, il commente ce qui est en train de se passer et lui demande de suivre ses instructions : "Maintenant applaudis, c'est la règle", dit-il avec un large sourire. »

(Carnet de terrain, séance du 14 décembre 2012)

Cédric vient parler à Déborah, nouvellement adhérente :

«Cédric : - Tu stresses encore?

Déborah :- Quand j'ai le guide je ne stresse pas, mais je ne sais pas ce qu'il faut faire, dis-moi, l'objectif c'était ça ?

Cédric : - Oui, est-ce qu'il a structuré son discours ? Est-ce qu'il a fait ça ?...Oui ? Donc conclusion, on a appris à le connaître... (il lit son évaluation) Oui, ça c'est bien utilisé... (il écrit son évaluation). »

(Carnet de terrain, séance du 24 janvier 2013)

Ces situations témoignent des inégalités de genre dans la prise en charge des adhérent·e·s, qui ne se limitent pas aux membres expérimenté·e·s. Ce point ressort particulièrement des observations ; s'il peut arriver aussi que des hommes nouvellement inscrits donnent des conseils à d'autres membres sur la base d'aptitudes professionnelles ou extra-professionnelles, dont ils se jugent porteurs, je n'ai jamais été témoin de situations analogues de la part des femmes. Kevin (43 ans, commercial) est arrivé dans le club à la même période que moi. Dans l'extrait qui suit, il me complimente, façon pour lui de manifester sa maîtrise et sa supériorité :

«Après mon premier discours, Mickaël et Kevin viennent me féliciter lors de la pause. Ils me serrent la main. Kevin met sa main sur mon épaule et me dit : "Tu vas voir, tu deviendras un grand orateur." Mickaël opine de la tête. »

(Carnet de terrain, séance du 7 février 2013)

On le voit à travers ces exemples, les interventions des hommes aguerris reposent sur une relation intersubjective forte : ils encouragent en se plaçant dans une position paternaliste. Leurs actions empreintes d'enthousiasme, d'empathie ou de complicité ne sont pas en elles-mêmes altruistes ou désintéressées. Elles correspondent aux pratiques du leader définies par l'association. En aidant les autres, ils ne font que se conformer aux qualités attendues des encadrant·e.s. Les extraits ci-dessus montrent que les hommes s'accommodent avec moins de difficulté à ces savoir-faire interactionnels. Par conséquent, si les compétences du leader reposent sur des présupposés attachés au genre féminin, nos observations ethnographiques montrent que dans le club étudié, leur apprentissage cadre en pratique davantage, mais de façon non explicitée, avec des compétences associées aux hommes : le sens du jeu et de la compétition, la capacité à prendre des initiatives, l'aisance relationnelle, etc. 
De surcroît, les savoir-faire interactionnels du leader sont interprétés par les membres masculins, qui lui associent une position de supériorité : « le leader gère... Il a l'autorité sans être autoritaire », pour reprendre une formule de Didier (51 ans, cadre commercial). Quand les hommes mobilisent en séance des figures de référence censées représenter leur vision du leader, il s'agit régulièrement d'hommes politiques américains. Que ce soit Obama ou, dans une moindre mesure, Al Gore ou Mitt Romney, tous sont considérés comme des modèles par les hommes de l'organisation. C'est ce que déclare Victorien (38 ans, dentiste/entrepreneur) à Jean (32 ans, coach) lors d'une discussion au terme d'une séance : « Eh ! Tu sais qu'Al Gore a fait l'association aux États-Unis ! C'est ce que laissent penser aussi les nombreux exercices d'improvisations qui consistent souvent à singer les manières d'être d'un politicien étasunien en s'imaginant à sa place : «Imagine que tu es Barack Obama, comment réagis-tu ? » De nombreuses phrases prononcées en cours de séance manifestent l'admiration des participant·e.s pour la compétence d'orateur, la « tchatche », l'optimisme, l'affabilité de ces personnages. Ces figures idéalisées ne cessent d'être opposées à certains hommes politiques français jugés « old school », comme Jacques Chirac ou Charles de Gaulle. Les Américains invoqués sont caractérisés par un fort souci d'afficher une posture amicale avec les citoyen'ne-s : mettre en scène sa vie privée, ses sentiments, exposer sa décontraction et sa sympathie ou encore chercher à divertir plus qu'à étayer un projet politique (ALMEIDA, 2003). Les opposer à d'anciennes figures françaises leur permet de rejouer l'opposition leader/patron, en soulignant cette fois plus explicitement la dimension genrée du leader. En public, ces personnalités n'hésitent pas à cultiver une certaine virilité de chef de famille et restent associées dans les médias à des notions correspondant aux représentations les plus communes du chef : maîtrise, charisme, courage, force, etc.

Alors que les compétences féminines sont souvent associées à la dimension relationnelle, les observations montrent que la conception du leader comme relationnelle et bienveillante se décline plus facilement au masculin dans le club. Les discours et les pratiques rejouent la connexion entre leadership et masculinité, mais cette fois dans une version chaleureuse, bienveillante, d'apparence plus égalitaire, qui tend à affaiblir les conduites masculines. En témoignent par exemple les railleries exprimées à l'égard d'Annie, cadre de santé qui fréquente l'association depuis sa promotion à l'hôpital il y a huit ans. Son style direct et ses évaluations sans ménagement contrastent avec l'esprit pacifié promu par l'association. Quand elle évalue des prises de parole, elle n'hésite pas à soumettre des conseils sans détour, de manière très directive : « Jérôme, va à l'essentiel et sois moins timide! Ta cartographie, elle est sympathique, mais ta conclusion est trop longue ! ; « Bravo Diane d'avoir recentré les impros ! »; « Sois moins timide, va voir un peu plus loin, viens les chercher davantage avec le regard! (sourires du public) »; " Kevin, quand tu donnes un support, on discute avec son voisin, et ton intérêt à toi, c'est que les gens te regardent du début jusqu'à la fin, okay ? (rires $d u$ public). » Annie fait rire ou sourire l'audience. Mais le lien entre le rire et l'irritation est paradoxal : si le rire autorise une libération à l'égard d'un souci ou d'un 
désagrément, il permet aussi, dans bien des cas, de signaler un acte jugé anormal dans une série d'actes considérés comme appropriés (FRISCH-GAUTHIER, 1961). Dans une association qui promeut une forme d'euphémisation des rapports d'autorité par une bienveillance associée à la féminité, Annie, par sa manière d'agir, trop « matrone »mot d'un des participants - porte atteinte à cette injonction. Sans lui faire le reproche directement ${ }^{16}$, des discussions informelles avec plusieurs membres font état de son autorité. Beaucoup la jugent trop professorale, autoritaire et directive, c'est-à-dire trop masculine, pour correspondre aux bonnes pratiques du leader.

Annie, la plus âgée avec Didier, est issue d'une famille moins dotée en capital culturel que les autres membres du club. Elle est moins diplômée et son univers professionnel fonctionne sur l'autorité et la hiérarchie, avec souvent de la violence verbale, des conflits ouverts et du sexisme assumé (ZolezIo, 2012), ce qui accentue le caractère hiérarchique de ses prises de parole. Dans le métier très féminisé qu'est celui d'infirmière, la promotion interne permet aux femmes d'accéder à des responsabilités hiérarchiques en devenant cadres intermédiaires. Cette fonction les place sous l'autorité le plus souvent d'hommes médecins et directeurs d'hôpitaux. Elles sont ainsi nombreuses à occuper une position d'autorité particulièrement inconfortable, soumise aux injonctions contradictoires d'un New Public Management, qui leur demande à la fois d'être la clé de voûte de l'édifice organisationnel et d'avoir davantage de compétences relationnelles (GADÉA, 2011). Dans les centres hospitaliers, où un nombre restreint de femmes occupe les postes de direction, ces femmes ont tendance à contrer les stéréotypes dont elles font l'objet en suivant l'exemple de leurs supérieurs masculins (KANTER, 1977 ; GUILLAUME, POCHIC, 2007). La longue expérience professionnelle d'Annie - de plus de trente ans - dans l'encadrement semble rendre difficile l'intégration de compétences dites «soft ». En somme, elle vient chercher dans ce club des qualités relationnelles plus adaptées aux transformations managériales de l'hôpital public, mais sa socialisation primaire et professionnelle se heurte aux socialisations des autres membres davantage liées aux relations commerciales.

16. Il m'est arrivé d'observer une évaluation qui faisait néanmoins transparaître explicitement ces reproches. Dans ce qu'il baptise « les qualités du discours », un membre pointe non sans ironie le ton professoral d'Annie : « Tu étais à la fois professeure de géographie, professeure d'histoire et enfin professeure d'histoire de l'art », phrase qui provoque le rire du public, en signe d'acquiescement. 


\section{Juger la communication : de la différence de classe dans la différence de genre}

\section{Évaluations des compétences en communication et durcissement des identités de genre}

À l'arrivée dans le club, ce sont les propriétés sociales spécifiques d'un·e invité.e qui le conduisent à adhérer ou non au dispositif. Les hommes comme les femmes justifient leur investissement par le désir de combler un déficit de ressources culturelles rencontré dans leur activité professionnelle. Pour ce faire, nous l'avons vu, l'évaluation est omniprésente et constitue même une ressource pédagogique essentielle : chaque membre qui effectue un discours devant le public est évalué. Un extrait du manuel numérique Évaluation efficace met en avant l'importance toute particulière de l'évaluation dans l'amélioration des leaders : « Les commentaires que font les membres d'un club à l'issue de la prestation d'un des leurs s'appellent une évaluation, qui constitue le fondement même de notre méthode de formation ». L'évaluation est ici ce qui donne le droit à des feedbacks, c'est-à-dire à des retours formulés par un membre, permettant à l'orateur ou l'oratrice/leader de recueillir des avis extérieurs sur sa prestation. Cette manière de faire n'est pas sans rappeler le monde de l'entreprise, en particulier les enquêtes d'évaluation et les entretiens de recrutement qui y ont cours. Dans le cadre professionnel, l'évaluation s'inscrit dans une quête de contrôle des incertitudes de la coordination, face à des exigences normalisées (MONCHATRE, 2011). Il s'agit bien souvent pour les salarié.e.s en situation d'orateur ou d'oratrice de convaincre, en alliant récit biographique, exercices de confiance en soi, d'éloquence et d'élocution. La mise en relation des critères d'évaluation des membres avec leurs caractéristiques personnelles fournit des indications sur les conceptions implicites associées à la notion de leader (CARTIER, 2001).

Dans une organisation où chacun·e, quel que soit son sexe, est tenu de présenter une image de soi dynamique, agréable et conciliante, et d'entretenir des relations pacifiées, les évaluations sont toujours l'occasion de mettre en œuvre une forme de psychologie ordinaire qui aboutit à interpréter, à travers une hexis corporelle et linguistique, des signes de compétence ou de personnalité. Les manières de parler et les postures sont interprétées au sein d'oppositions symboliques issues des injonctions de l'association. Les termes « mou », « timide » (opposés à « dynamique », « à l'aise ») structurent les évaluations plus ou moins élogieuses. Les membres reprennent en règle générale les termes de ces oppositions pour exprimer le caractère évident des différences entre hommes et femmes. En atteste une première évaluation de Mickaël (25 ans, sans emploi), inscrit depuis deux ans au club :

« Idriss [avocat de formation, 35 ans], je t'ai trouvé très à l'aise, dynamique. Tu es venu, t'as bien pris l'espace, tu étais sûr de toi, très à l'aise [...]. Cédric, tu étais aussi à l'aise, tu n'as pas fait d'hésitation, en termes de regard, tu regardais bien la salle. Par contre avec tes gestes, tu as un peu tendance à faire la savonnette (mimant avec 
ses bras). C'est dommage parce que le reste, c'était bien (sourire), on sent que tu es un vrai ministre ! [...] Déborah toi, tu es arrivée pleine d'humour, donc ça c'était très bien. Par contre, dès que tu étais là (signes de bras pour montrer la scène), tu ne savais pas la suite et c'est tout de suite redescendu et je t'ai sentie hésitante et timide [...]. Ensuite, Olivier [commercial, 31 ans], pareil : c'était très dynamique [...] ; comme point d'amélioration, tu étais un peu trop statique tout de même. »

(Carnet de terrain, séance du 25 avril 2013)

Les évaluations de ce type ne sont pas l'apanage des hommes. Les femmes aussi sont en position d'évaluatrices et utilisent les mêmes oppositions. Premier extrait avec Déborah (42 ans, employée de banque), second extrait avec Audrey (34 ans, assistante commerciale) :

Déborah : «Audrey, tu as commencé de manière hésitante, peut-être, mais après tu t'es approprié ton sujet et tu étais vraiment convaincue par ce que tu disais. Je trouve aussi que tu as tendance à rester un peu renfermée [...]. À l'opposé Mickaël, tu occupes très bien l'espace, tu captais ton auditoire, on était comme obligé de t'écouter. Évelyne, tu as un humour assez doux, j'aime [...]. Boris, toi aussi, tu es super à l'aise, donc l'improvisation était très bien développée [...] ; j' ai beaucoup aimé également, c'est ta prise de risque. »

(Carnet de terrain, séance du 28 février 2013)

Audrey : «Yannick, j'ai bien aimé ton discours, tu vas directement à l'essentiel, introduction, problématique, annonce du plan, annonce d'une façon très directe $[\ldots]$; tu étais à l'aise également [...] ; j'ai beaucoup aimé ton originalité, ton humour, mais j'aurais préféré davantage de structure au niveau du temps. Sinon, très beau discours. »

(Carnet de terrain, séance du 28 février 2013)

Les descriptions d'évaluations montrent à quel point les performances de genre sont traitées de manières différentes. Les hommes considérés comme de mauvais communicants sont associés aux termes «mou », « statique », alors que des femmes jugées négativement sont plutôt associées aux termes «timide », « hésitante » ou « renfermée ». Du reste, quand il s'agit cette fois de critiques positives, les hommes sont systématiquement « à l'aise », « dynamiques », dotés de qualités d'initiative, ils sont « directs » et prennent des « risques », ce qui est plus inhabituel pour les femmes.

\section{Le jugement du langage et de l'hexis comme renforcement des rapports de classe}

Selon Bourdieu, qui réutilise les analyses du sociolinguiste William Labov, le rapport au langage des classes « à bonne volonté culturelle » se caractérise par la propension à l'hypercorrection, c'est-à-dire par la tendance à essayer d'être plus correct qu'il ne faut, et à rajouter en correction (BouRDIEU, 2001). Ceci se révèle avec acuité lors des séances, les évaluateurs faisant toujours preuve d'une très grande exigence. 
Première intervention de Carole (36 ans, juriste), adhérente régulière, puis évaluation d'Éric, cadre d'une trentaine d'années, nouveau dans le club :

Carole : « Alors, Audrey, dans ton évaluation tu as dit “j'l'a trouve pas"; peut-être "je ne la trouve pas" pour plus de fluidité tout simplement. Un moment, tu as prononcé train "twouin" aussi (rires légers de l'audience). Leo, dans ton improvisation, tu as dit "procédons, je vous prie, de la manière suivante", ce qui était très, très bien dit. Olivier, tu as dit "protagoniste", très bien ; mais tu as aussi utilisé le mot "super" pour dire "super terre à terre" en parlant de la conclusion de Cédric, utilise plutôt le mot "quelconque" ».

(Carnet de terrain, séance du 7 février 2013)

Éric : « Déborah, tu as dit "fringue", c'est plus vêtement ; par contre, tu as utilisé "allègrement", ce qui est très joli. Didier [...], à un moment, t'as dit "comme si ça t'était arrivé à toi", j'aurais préféré : "Comme si cela t'était arrivé." (rires de l'audience)»

(Carnet de terrain, séance du 10 janvier 2013)

Les élisions orales «j'l'a trouve pas » ou «ça t'était arrivé à toi », les erreurs de prononciation et l'usage de mots familiers sont corrigés avec rigueur. Les évaluateurs se réfèrent au langage formel, voire soutenu, requis dans tous les espaces institutionnels, que ce soit l'école, l'administration publique ou bien l'entreprise. Les adhérent.e.s qui invoquent ce langage ne prennent guère en compte le fait qu'il existe des conditions sociales de formation et de matérialisation des pratiques oratoires ; les orateurs/leaders tels Obama, Romney ou Al Gore ont la propriété de cumuler les attributs sociaux les plus valorisés - ce sont des hommes, hétérosexuels, pères de famille - et les capitaux et certifications universitaires les plus légitimes - tous trois sont très fortunés et diplômés de l'université d'Harvard. Ce qui conduit les membres à mieux évaluer les participant·e.s venant de milieux favorisés, en amoindrissant et parfois même en neutralisant les handicaps liés au genre selon les dispositions sociales de l'évalué·e. C'est ce que montre l'intervention d'Olivier, commercial, diplômé d'une petite école de commerce et adhérent depuis trois mois, qui évalue Carole (36 ans, juriste en entreprise). Carole manifeste une grande aisance pour s'exprimer en public. Contrairement aux autres membres vis-à-vis desquels Olivier est très critique, il juge sa prestation « convaincante », « très claire » et apprécie particulièrement sa « gestuelle » et ses « déplacements ». Il utilise à son propos l'expression « à l'aise », qu'il réserve par ailleurs le plus souvent aux hommes.

Un autre extrait de séance a particulièrement retenu mon attention. Il s'agit d'une évaluation de Didier (51 ans, cadre commercial) par Boris (33 ans, chef de projet informatique), devant les adhérent·e.s :

« Didier, ton objectif était d'être un leader persuasif. Alors... je ne doute pas que tu sois un leader (sourire de quelques membres)... et que tu sois persuasif également (rires sourds du public), mais ce que l'on voulait voir ce soir dans ce discours, c'est comment tu pouvais le démontrer à travers ce discours (sourire de Boris en direction 
de Didier) [...] Ton aisance, maintenant. Moi qui t'ai connu tout timide, je trouve que tu as bien progressé. Tu es maintenant plus calme, plus posé, plus sobre [...]. Ça fait plusieurs séances que je commence à te connaître Didier... Je vais résumer la chose en disant qu'en fait Didier, tu es un poète (sourire de Boris et rires $d u$ public) et, comme tous les poètes, tu es difficile à lire (rires du public). Sauf que le problème, c'est que ce ne sont pas des clients... (souriant et montrant l'audience avec les bras).»

(Carnet de terrain, séance 4 décembre 2012)

On retrouve ici la correction de l'hexis corporel traitée comme un révélateur de qualités personnelles. Si Didier est jugé moins « timide » qu'avant, il est qualifié de « calme » et de « sobre », ces deux adjectifs plutôt utilisés d'habitude pour les hommes tendent à signifier qu'il ne s'est pas pour autant réellement amélioré ; le bon leader étant considéré comme relationnel et dynamique. Les rires de l'audience s'analysent doublement : ils soulignent la forme non conventionnelle des critiques, plutôt directes, tout en établissant une complicité évidente avec les remarques de l'évaluateur. Cette scène acte la supériorité de Boris, bien meilleur leader que Didier.

Boris est né en Martinique, d'une mère secrétaire médicale et d'un père médecin urgentiste. Au cours de l'entretien, il tient un langage fortement inspiré du jargon managérial. Diplômé de l'École centrale de Paris, il est membre de la structure depuis sept ans. Peu après l'obtention de son diplôme de fin d'études, il est invité par un ami de son père, également médecin, à une semaine de séminaire de développement personnel à Londres « avec un coach très réputé ». À cette époque, il souffre d'un manque de confiance en lui et de stress pour parler en public. C'est à la suite de cette première expérience, décrite comme très positive, qu'il découvre l'association Becomealeader et y adhère aussitôt. Aujourd'hui ingénieur, il est considéré par tous comme un communicant et un leader accompli. Au moment des pauses, Boris est constamment appelé pour trancher des questions ou répondre aux remarques des nouveaux et nouvelles membres :

«Une invitée désire avoir de l'aide à propos d'indications peu claires dans le manuel de communication. Elle s'approche de Victorien. Il lui répond ne pas comprendre et l'oriente vers Boris : "il saura...", finit-il. Boris arrive, la nouvelle adhérente lui montre la partie du manuel posant problème. Il regarde le document et lui donne raison : "Ah, ils ont trop mal traduit... Bon, ne t'occupe pas de ça et passe à l'objectif suivant." »

(Carnet de terrain, séance 4 décembre 2012)

Cette séquence montre bien l'autorité que les membres reconnaissent à Boris, qui décide en dernier ressort d'outrepasser les règles du manuel. Sa situation contraste avec celle de Mickaël, né en région parisienne de parents petits commerçants, un des plus jeunes membres réguliers du club. Il souhaite chercher du travail dans la publicité ou fonder une start-up après des études en communication à l'université. N'ayant occupé que des petits boulots pour financer ses études, il considère sa formation au leadership comme une expérience valorisant son curriculum vitae. Par rapport aux 
autres, il détonne par ses façons non conventionnelles de parler, parfois argotiques, et son manque d'articulation. À la fin d'une séance, Mickaël me confie avec amertume son insatisfaction. Il déclare ne pas arriver, comme Boris ou Victorien, «à avoir la banane [...] ; c'est ça un leader, c'est celui qui a tout le temps la banane !». Les prénoms cités indiquent que seuls les hommes du club sont concernés par cette affirmation. L'appréciation de Mickaël renvoie à l'idée que le maintien d'une allure réjouie, constitutif de l'image du leader, est inégalement réparti selon le sexe et la position sociale. Si c'est une pratique aujourd'hui facile pour Boris, elle demeure pénible pour lui. Il nous semble avec Mickaël, et dans une moindre mesure avec Annie, que les normes du leader relationnel et agréable déstabilisent particulièrement les membres d'origine plus populaire.

La composition sociale du club, davantage constituée de salariée·s non manuel·le·s et d'encadrant-e.s des premiers niveaux hiérarchiques, invite à prendre en compte la pluralité des échelons dans la capacité d'adoption des discours managériaux et donc à en relativiser l'emprise sur tous les salarié.e.s. Ces professionnel-le·s, dans l'attente d'une meilleure situation, expriment un vif attachement au paradigme gestionnaire. Le dispositif de formation que constitue l'association analysée se révèle être pour eux et pour elles à la fois un instrument de mise en conformité avec les exigences managériales et un lieu où ils et elles espèrent trouver des savoirs sélectifs décisifs pour progresser dans leur carrière - parler en public avec assurance, avoir de l'entregent, etc.

Cependant, une fois que les membres sont entré-e.s dans le club, ces savoir-faire se redéfinissent et tendent à retrouver les principes de domination de genre et de classe. C'est ainsi que contrairement à ce que promeuvent les théoricien.ne.s du «nouveau capitalisme » et les prophéties associées à un management au féminin, l'apprentissage de ces savoir-faire ne semble pas à même d'améliorer les chances de promotion des femmes. En effet, l'apprentissage reproduit les hiérarchies socio-sexuées qui favorisent les hommes issus de la partie haute des classes moyennes.

Nos observations montrent que la valorisation de l'entregent et de la communication chez les adhérent.e.s du club requiert des compétences plus souvent valorisées et développées par les hommes - le sens du jeu et de la compétition, la capacité à prendre des initiatives, l'aisance relationnelle, etc. - mais dans une version euphémisée, mobilisée à des fins bienveillantes, comme l'aide des nouveaux et des nouvelles adhérent.e.s. Les appropriations différenciées du dispositif conduisent ainsi à conforter l'ordre scolaire et sexuel existant en flattant les membres les plus doté.e-s en capital culturel et, inversement, en amenant à dénigrer voire à discréditer les attributs sociaux déjà dévalorisés sur le marché du travail. Sans fournir un changement dans l'ordre social ni une potentielle subversion du système de genre, l'apprentissage relationnel des hommes contraste en revanche avec les modèles de virilité plus traditionnels. Il 
suscite l'affaiblissement des relations de domination sans pour autant participer à la renégociation de leur pouvoir.

On peut raisonnablement penser que ces effets, analysés lors de séances du club, perdurent en situation de travail : les prises de parole jouent un rôle central en entreprise. Il est néanmoins difficile de le savoir tant ces derniers sont par ailleurs contraints par les enjeux productifs et hiérarchiques propres aux organisations. D'autres investigations seraient certainement bienvenues à cette fin.

\section{BibliogRAPHIE}

Amintas A., Junter A. (2009), «L'égalité prise au piège de la rhétorique managériale », Cahiers du genre, $\mathrm{n}^{\circ} 47$, pp. 103-122.

BERENI L. (2011), «Le discours de la diversité en entreprise : genèse et appropriations », Sociologies Pratiques, n ${ }^{\circ} 23$, pp. 9-19.

BERNARD L. (2012), «Le capital culturel non certifié comme mode d'accès aux classes moyennes. L'entregent des agents immobiliers », Actes de la recherche en sciences sociales, $\mathrm{n}^{\circ}$ 191-192, pp. 68-85.

Borzeix A., Charles J., Zimmermann, B., (2015), « Réinventer le travail par la participation. Actualité nouvelle d'un vieux débat », Sociologie du travail, n 57, pp. 1-19.

Billing Y.D., Alvesson M. (1989), "Four Ways of Looking at Women and Leadership ", Scandinavian Journal of Management, $\mathrm{n}^{\mathrm{0}}$ 1, pp. 63-80.

BLANCHARD S. (2010), « Café amer et égalité sucrée : l'accès des femmes aux postes à responsabilité à travers le prisme de la rhétorique patronale », Recherches Féministes, 23-2, pp. 165-182.

Blanchard S., Boni-Le Goff I., Rabier M. (2013), « Une cause de riches ? L'accès des femmes au pouvoir économique », Sociétés contemporaines, n ${ }^{\circ}$ 89, pp. 101-130.

Boltanski L., Chiapello E. (1999), Le nouvel esprit du capitalisme, Paris, Gallimard.

BourdiEu P. (2001), Langage et pouvoir symbolique, Paris, Seuil.

Calmand J., ÉPIPHAne D., Hallier P. (2009), « De l'enseignement supérieur à l'emploi : voies rapides et chemins de traverse. Enquête "Génération 2004", interrogation 2007 », Emploi Formation, CEREQ, n ${ }^{\circ} 43$.

CARTIER M., (2001), « Nouvelles exigences dans les emplois d'exécution des Services publics », Genèses, nº42, pp. 72-91.

COHEN Y. (2013), Le siècle des chefs. Une histoire transnationale du commandement et de l'autorité (1890-1940), Paris, Éditions Amsterdam.

D’Almeida F. (2003), « L'américanisation de la propagande en Europe de l'Ouest (19452003 ) », Vingtième Siècle. Revue d'histoire, nº 80, pp. 5-14.

FRISCH-GAUTHIER J. (1961), « Le rire dans les relations de travail », Revue française de sociologie, 2-4, pp. 292-303. 
GAdÉA C. (2011), « Cadres de santé, le chainon manquant », in Bouffartigue P., Gadéa C., Pochic S., Cadres, classes moyennes, vers l'éclatement, Paris, Armand Colin.

Goffman E. (1973), La Mise en scène de la vie quotidienne, I : La présentation de soi, Paris, Éditions de Minuit.

Goux D., Maurin E. (2012), Les Nouvelles classes moyennes, Éditions du Seuil, « La république des idées», Paris.

Guillaume C., Pochic S. (2007), « La fabrication organisationnelle des dirigeants. Un regard sur le plafond de verre », Travail, genre et sociétés, $\mathrm{n}^{\mathrm{0}}$ 17, pp. 79-79.

INSEE (2017), « Femme et homme, l'égalité en question ».

KANTER M. R. (1977), Men and Women in the Corporation, Basic Books, New York.

Karvar A., Rouban L. (2004), Les Cadres au travail. Les nouvelles règles du jeu, La Découverte, Paris.

LAUFER J. (2005), « La construction du plafond de verre : le cas des femmes cadres à potentiel », Travail et Emploi, $\mathrm{n}^{\circ} 102$, pp. 31-44.

LAUFER J., (2009), « L'égalité professionnelle entre les hommes et les femmes est-elle soluble dans la diversité ? », Travail, genre et sociétés, n $^{\circ} 21$, pp. 29-54.

LinHART D. (2003), « Organisation du travail et participation des salariés » in Allouche, J. (Ed.), Encyclopédie des ressources humaines, Vuibert, Paris, pp. 1067-1075.

MARRY C. (2001), « La féminisation de la profession d'ingénieur en France et en Allemagne », in Bouffartigue Paul (dir.), Les Cadres, la grande rupture, Paris, La Découverte, pp. 281-296.

Monchatre S. (2011), « Ce que l'évaluation fait au travail. Normalisation du client et mobilisation différentielle des collectifs dans les chaînes hôtelières », Actes de la Recherche en sciences sociales, $\mathrm{n}^{\circ} 189$, pp. 42-57.

Pailhé A., Solaz A. (2010), « Concilier, organiser, renoncer : quel genre d'arrangements ? », Travail, genre et sociétés, $\mathrm{n}^{\circ} 24$, pp. 29-46.

Pochic S. (2005), « Faire carrière : l'apport d'une approche en termes de genre », Formation Emploi, n ${ }^{\circ}$ 91, pp. 75-93.

SALMAN S. (2014), « Un coach pour battre la mesure ? La rationalisation des temporalités de travail des managers par la discipline de soi », Revue d'anthropologie des connaissances, $\mathrm{n}^{\circ} 8$, pp. 97-97.

Scotт W. J. (1986), “Gender : A Useful Category of Historical Analysis”, The American Historical Review, n ${ }^{\circ}$ 5, pp. 1053-1075.

SteVens H. (2012), « Autonomie récusée, autonomie fabriquée. Informaticiens à l'épreuve de l'Entreprise de Soi », Genèses, nº 87, pp. 90-112.

SteVens H. (2013), « Entre émancipation symbolique et reproduction sociale », Travail et Emploi, $\mathrm{n}^{\circ}$ 133, pp. 39-51.

Zolesio E. (2012), Chirurgiens au féminin? Des femmes dans un métier d'hommes, « Le Sens social », Rennes. 